\title{
Management of Maxillofacial Osteosarcomas in Kenya
}

\author{
Symon Guthua, Martin Kamau, Nicholas Abinya
}

University of Nairobi

Correspondence to: Dr. Martin Kamau, PO Box 19676-00202, Nairobi, Kenya; email: mrtnkamau@yahoo.com

\begin{abstract}
Background: Though not common, maxillofacial osteosarcomas present a unique challenge in management due to a multitude of factors, such as difficulty in diagnosis and local complex anatomy, making surgical excision difficult as well as debate necessary on the usefulness of adjunct treatment modalities, such as chemotherapy and radiotherapy. Nonetheless, osteosarcomas are a significant health burden because of their high morbidity and mortality. Method: Retrospective cross-sectional study of records archived in the School of Dental Sciences, University of Nairobi. Results: 25 cases of maxillofacial sarcomas were seen over 26 years. Mean age of occurrence was 35.68 years with a preponderance for females (17 cases). The mandible was the most affected site, accounting for 18
\end{abstract}

cases. Discussion: The management of maxillofacial sarcomas in our setting presents significant challenges arising from multiple factors such as lack of standardised treatment protocol, late presentation of patients, diagnostic challenges and loss to follow-up.

Keywords: Maxillofacial osteosarcomas, Management protocol, Kenya

Ann Afr Surg. 2020; 17(1):26-29.

DOI: http://dx.doi.org/10.4314/aas.v17i1.7

Conflicts of Interest: None

Funding: None

(C) 2020 Author. This work is licensed under the Creative Commons Attribution 4.0 International License.

\section{Introduction}

With an incidence of 1:100,000 (1,2), osteosarcoma is the most common primary neoplasm of bone. Most head and neck sarcomas are of the soft tissue type with only $20 \%$ being of bony or cartilaginous origin (3). In the USA, it is estimated that less than $4 \%$ of all recorded osteogenic sarcomas occur in the jaw (4). The mandible and maxilla are the predominant sites of head and neck osteosarcoma (HNOS), although extragnathic bone as well as soft tissue sites may be affected [6]. The 5-year disease-specific survival rate for patients with HNOS has been poor with most studies reporting survival rates of $23-37 \%$, with maxillary tumours showing worse prognosis (5-8). Maxillofacial sarcomas have a biological behaviour that differs from that of the long bones: the average age of onset is 10-20 years later than their skeletal variants, distant metastases are rarer and survival rates are more favourable (9). The mainstay of treatment for osteosarcoma of the jaws is complete surgical excision with free margins as well as reconstruction to improve function and quality of life post-treatment. The use of multidrug chemotherapy (both neoadjuvant and adjuvant) in the management of skeletal OS is well established with notable improvements in survival rates, but the same cannot be said for OS in the maxillofacial

region. Two meta analyses reviewing the outcomes of adding chemotherapy regimes in head and neck OS showed conflicting results (10). Nonetheless, several individual centre reports have shown a benefit with the addition of neoadjuvant or adjuvant chemotherapy, or both (11). In the maxillofacial region, extensive lesions may provide a challenge where the complex local anatomy and proximity to vital structures may preclude wide excision with adequate tumour-free margins.

This paper reviews 25 cases of OS seen over 26 years in one specialized department in a teaching institution.

\section{Materials and Methods}

The study was conducted at the Department of Oral and Maxillofacial Surgery, School of Dental Sciences, University of Nairobi, using patient clinical and histopathologic records archived at the department. This was a retrospective cross-sectional study analysing all the records of patients who had a histologic diagnosis of maxillofacial OS. Patient clinical and histopathologic records were retrieved and data recorded in a data collection form. Incomplete patient records were excluded from the study. Ethical approval was obtained from the Ethics, Research and Standards Committee of the Kenyatta National Hospital and the University of 
Nairobi (approval number: P170/6/2009).

\section{Results}

Twenty-five cases were analysed, and women were more affected ( 17 cases) than men ( 8 cases). The mandible was the most common site afflicted (18 cases) while 7 cases were in the maxilla. The age range was from 1 week old to 70 years; 15 patients were below the age of 39 years. The mean age of occurrence was 35.68 years. Table 1 shows the distribution of cases by age.

Table 1. Distribution of cases by age

\begin{tabular}{cc}
\hline Age group (years) & Osteosarcoma cases \\
\hline $0-9$ & 1 \\
$10-19$ & 4 \\
$20-29$ & 6 \\
$30-39$ & 4 \\
$40-49$ & 3 \\
$50-59$ & 3 \\
$>60$ & 4 \\
\hline
\end{tabular}

The histopathologic subtypes varied between osteoblastic, fibroblastic and chondroblastic highgrade variants. One patient was diagnosed with an aggressive telangiectatic/vascular type of osteosarcoma while another had a discordant diagnosis: initial histological diagnosis was osteoblastoma, later revised to osteosarcoma after review by a panel of pathologists. Musculoskeletal Tumour Society Cancer Centre staging was noted for 20 patients only who were in the surgery and chemotherapy group and the palliative chemoradiotherapy group (3). The patients were all in stage II. No staging was noted for five patients who had been treated earlier in this series with either surgery alone or surgery and radiotherapy. No details were noted from the clinical records of why these patients were offered these treatment modalities.

Over the past 26 years, the modalities of management have mainly been multimodal therapy involving

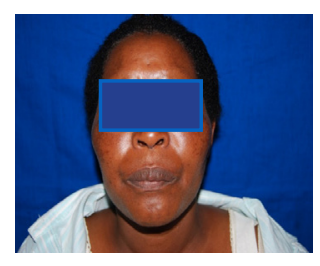

Figure 1. Above, extraoral appearance; right, intraoral appearance at presentation.

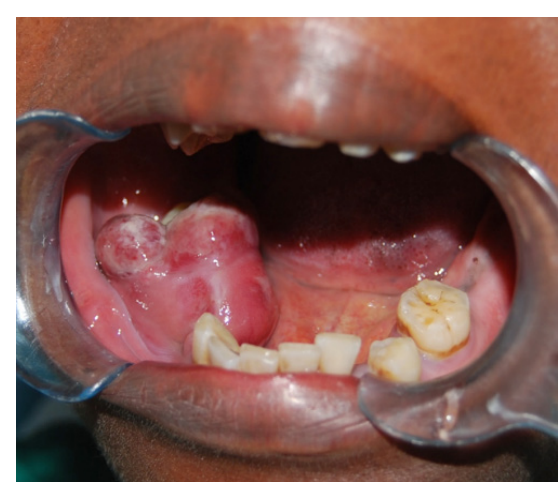

neoadjuvant chemotherapy, then cytoreduction therapy followed by postoperative adjuvant chemotherapy (Table 2).

Table 2. Treatment modalities and outcomes

\begin{tabular}{|c|c|c|}
\hline Treatment modality & $\begin{array}{l}\text { No. of } \\
\text { patients }\end{array}$ & Outcome \\
\hline Surgery alone & 4 & $\begin{array}{l}\text { Unknown-All lost to } \\
\text { follow-up posttreatment }\end{array}$ \\
\hline $\begin{array}{l}\text { Surgery + } \\
\text { chemotherapy } \\
\text { (neoadjuvant and } \\
\text { adjuvant) }\end{array}$ & 13 & $\begin{array}{l}5 \text { patients still on follow- } \\
\text { up } 10 \text { years. Stable } \\
\text { posttreatment }\end{array}$ \\
\hline $\begin{array}{l}\text { Surgery + } \\
\text { radiotherapy }\end{array}$ & 1 & $\begin{array}{l}\text { Unknown-Lost to } \\
\text { follow-up posttreatment }\end{array}$ \\
\hline $\begin{array}{l}\text { Palliative chemo- } \\
\text { radiotherapy }\end{array}$ & 7 & All deceased \\
\hline
\end{tabular}

\section{Case - Mandibular osteosarcoma}

Patient MM, a 54-year-old female, was referred with a swelling of the right side of the mandible of one month duration (Fig. 1). She had impaired sensation of the right inferior alveolar nerve. There was no significant medical history apart from hyperacidity related to non-steroidal anti-inflammatory drugs.

A CT scan revealed a lytic soft tissue mass arising from the right mandibular alveolus and extending to the surrounding soft tissues (Fig. 2).

Histopathology sections (Fig. 3) showed features of osteoblastic type (high grade) osteosarcoma.

High-resolution chest CT scan (Fig. 4) confirmed there was no metastasis of the tumor to the chest.

Management protocol for the patient included multimodal treatment as follows:

- neoadjuvant chemotherapy-3 cycles

- surgery (postchemotherapy) and initial primary reconstruction-tumour specimen was resected enbloc and reconstructed with a $2.5 \mathrm{~mm}$ preformed titanium plate and soft tissue (cheek and floor of mouth) augmentation with a pectoralis major
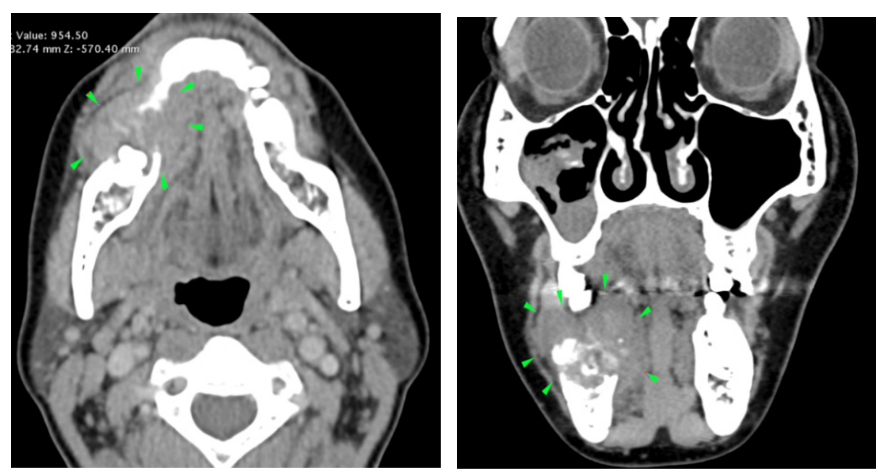

Figure 2. Left, CT scan - axial view; right, coronal view. The mass is marked in green arrows. 
pedicled flap (Fig. 5).

- postoperative chemotherapy-4 cycles after 6 weeks postoperative (Fig. 6).

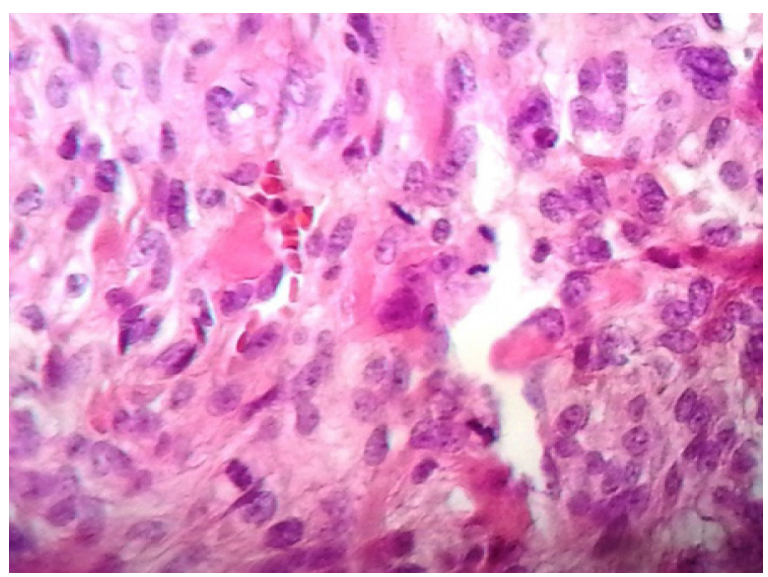

Figure 3. Histopathology specimen.

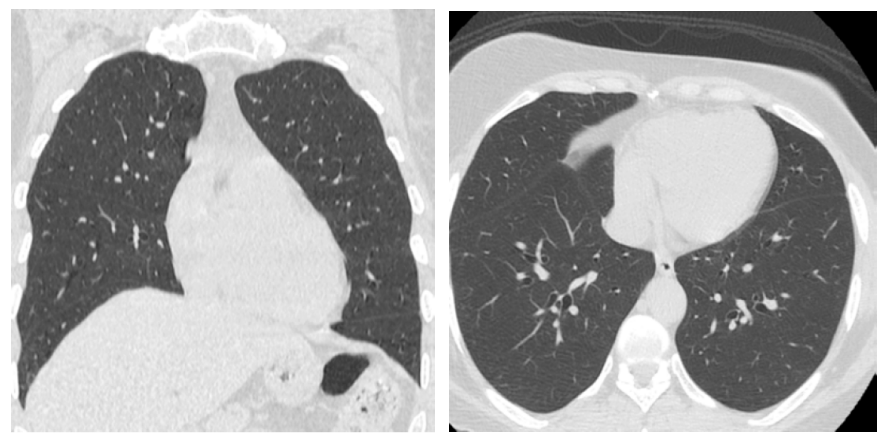

Figure 4. High resolution chest CT scan.
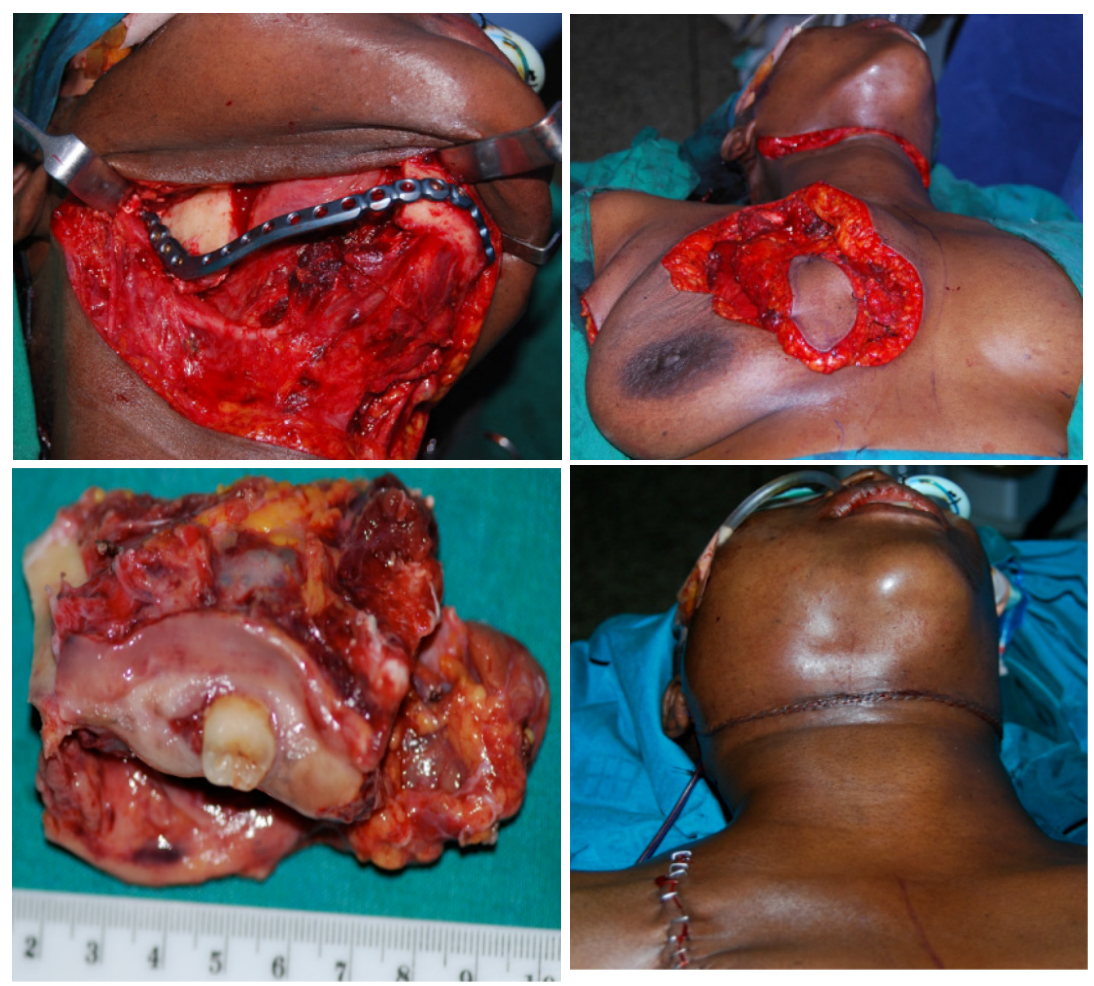

Figure 5.Clockwise: Titanium reconstruction plate, pectoralis major flap with skin paddle for intraoral closure, immediate postoperative, tumour specimen after resection.
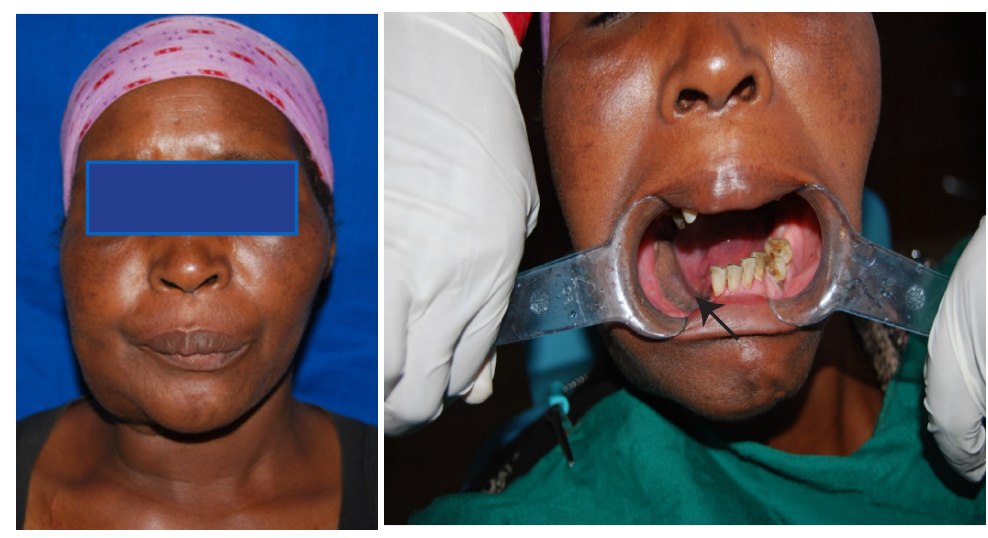

Figure 6. Left, extra oral appearance; right, intraoral appearance. Note the pectoralis major skin paddle (arrow).

\section{Discussion}

Generally, maxillofacial mesenchymal malignancies are rare. In the present study, 25 maxillofacial osteosarcomas were noted over 26 years. Some trends were noted in the demographic distribution of the maxillofacial sarcomas. In our study, the most frequent site of occurrence was the mandible, which concurs with most of the reports in literature $(1,3)$. Analysis of age distribution of maxillofacial osteosarcomas in this study showed that the mean ages at first presentation of maxillofacial osteosarcoma was consistent with data from other African studies (12).

The lower mean age of occurrence of osteosarcoma is probably influenced by the lower age expectancy in the Kenyan population (52 years in 2006) (12). Gender distribution showed a female preponderance, in contrast to literature which did not note any real preference for any gender $(1,2,12)$.

The management of osteosarcoma remains a challenge. First, these tumours remain relatively rare and may be considered as "orphan diseases" (13). In our case series we encountered 25 cases over 26 years. Second, they remain a challenge to diagnose. Our series did have a case that was initially diagnosed as an osteoblastoma. The clinical behaviour of the lesion did not correspond with the histological examination. Tissue specimen was re-evaluated and a diagnosis of osteosarcoma was made. Finally, management of the tumours presents challenges due not only to their aggressive nature but also to a lack of consensus on the mode of management. Literature on the management of maxillofacial 
sarcomas is scarce, and the little that is available does not provide consensus on the treatment protocol. While aggressive surgical extirpation is necessary, the use of chemotherapy in maxillofacial osteosarcomas remains controversial $(5,10)$. The use of chemotherapy in managing appendicular (long bone) osteosarcoma is well proven, but evidence is conflicting on its use in the head and neck (11). Nevertheless, it appears the use of chemotherapy has some advantage over surgery alone (4). Radiotherapy is not as effective as chemotherapy as osteosarcomas have been shown to be radio-resistant. The five patients in this study who remained disease free for 10 years had gone through a management approach involving neoadjuvant chemotherapy (3 cycles), followed by complete surgical extirpation and then adjuvant chemotherapy (4 cycles). From our case series, the overall outcomes were relatively poor with all patients presenting in stage III and this may have been attributed to late presentation of patients with these aggressive lesions. A challenge still remains of patient follow-up in our setting. Of the 25 cases in our case series, 12 patients were lost to follow-up possibly due to socio-economic factors, 7 patients were deceased and 5 patients are still on follow-up (10-year median time). Management of sarcomas remains a challenge essentially because these cancers are rare, they are biologically diverse and difficult to diagnose, a large multicentre evidence base for treatment is lacking, treatment regimens are complex and intense, and they have a high mortality. The National Cancer Control Network (US) and the National Institute for Health and Clinical Excellence (UK) have developed detailed recommendations limiting management of sarcomas to MDT sarcoma teams with specialist sarcoma expertssurgeons, pathologists, radiologists, oncologists—as well as support staff (14).

\section{Conclusion}

The management of jaw sarcomas in Kenya remains a challenge. Possibly, embracing the sarcoma centre model would improve outcomes by concentrating patient load and expertise in these centres with the attendant advantages of long-term follow-up and development of evidence-based treatment protocols.

\section{Acknowledgements}

The authors thank the staff of the Department of Oral and Maxillofacial Surgery, School of Dental Sciences, University of Nairobi, for their support.

\section{References}

1. Sturgis E, Potter B. Sarcomas of the head and neck region. Curr Opin Oncol. 2003; 15:239-52.

2. Garrington G, Scofield H, Cornyn J, et al. Osteosarcoma of the jaws: Analysis of 56 cases. Cancer. 1967; 20:377-91.

3. Rapidis A. Sarcomas of the head and neck in adult patients. Expert Rev Anticancer Ther. 2008; 8(8):1271-97.

4. August M, Magennis P, Dewit D. Osteogenic sarcoma of jaw: Factors influencing prognosis. Int J Oral Maxillofacial Surg. 1997; 26:198-204.

5. Kassir R, Rassekh C, Kinsella J, et al. Osteosarcoma of the head and neck: Meta-analysis of nonrandomized studies. Laryngoscope. 1997; 107:56-61.

6. Caron A, Hajdu S, Strong E. Osteogenic sarcoma of the facial and cranial bones: A review of forty-three cases. Am J Surg. $1971 ; 122: 719-25$.

7. Philip T, Iliescu C, Demaille M, et al. High-dose methotrexate and HELP-doxorubicin in non-metastatic osteosarcoma of the extremity: A French multicentre pilot study. Ann Oncol. $1999 ; 10: 1065-71$.

8. Ferrari S, Mercuri M, Picci P, et al. Non-metastatic osteosarcoma of the extremity: Results of a neoadjuvant chemotherapy protocol IOR/OS-3) with high-dose methotrexate, intraarterial or intravenous cisplatin, doxorubicin, and salvage chemotherapy based on histologic tumor response. Tumori. 1999; 85:458-64.

9. Russ J, Jesse R. Management of osteosarcoma of the maxilla and mandible. Am J Surg. 1980; 140:572-6.

10. Smeele L, Kostense P, van der Waal I, et al. Effect of chemotherapy on survival of craniofacial osteosarcoma: A systematic review of 201 patients. J Clin Oncol. 1997; 15:363-7.

11. Laskar S, Basu A, Muckaden M, et al. Osteosarcoma of the head and neck region: Lessons learned from a singleinstitution experience of 50 patients. Head Neck. 2008; 30:1020-6.

12. Kamau M, Chindia M, Dimba E, et al. Clinico-histopathologic types of maxillofacial malignancies with emphasis on sarcomas: A 10-year review. East Afr Med J. 2011; 88:19-27.

13. Orphanet Report Series: List of rare diseases and synonyms listed in alphabetical order. http://www.orpha.net/ orphacom/cahiers/docs/GB/List of rare diseases in alphabetical order.pdf. 2016

14. National Institute for Health and Clinical Excellence: Improving outcomes for people with sarcoma. NICE guidance on cancer services. 2006. 